\title{
Delivery of tumor-homing TRAIL sensitizer with long-acting TRAIL as a therapy for TRAIL-resistant tumors
}

Yumin Oh, ${ }^{\mathrm{a}, \mathrm{b}, 1}$ Magdalena Swierczewska, ${ }^{\mathrm{a}, \mathrm{b}, 1}$ Tae Hyung Kim, ${ }^{\mathrm{a}, \mathrm{b}}$ Sung Mook Lim, ${ }^{\mathrm{c}}$ Ha Na Eom,

Jae Hyung Park, ${ }^{\mathrm{d}}$ Dong Hee Na, ${ }^{\mathrm{e}}$ Kwangmeyung Kim, ${ }^{\mathrm{f}}$ Kang Choon Lee, ${ }^{\mathrm{c}}$ Martin G. Pomper, ${ }^{\mathrm{a}, \mathrm{g}}$ and Seulki Lee ${ }^{\mathrm{a}, \mathrm{b}, \mathrm{g}, *}$

${ }^{\mathrm{a}}$ Russell H. Morgan Department of Radiology and Radiological Science and ${ }^{\mathrm{b}}$ Center for Nanomedicine at the Wilmer Eye Institute, Johns Hopkins School of Medicine, Baltimore, Maryland, USA. ${ }^{\mathrm{c}}$ College of Pharmacy and ${ }^{\mathrm{d}}$ Department of Polymer Science and Engineering, Sungkyunkwan University, Suwon, Korea. ${ }^{\mathrm{e}}$ College of Pharmacy and Research Institute of Pharmaceutical Sciences, Kyungpook National University, Daegu, Korea. ${ }^{\mathrm{f}}$ Center for Theragnosis, Korea Institute of Science of Technology, Seoul, Korea. ${ }^{\mathrm{g}}$ Department of Materials Science and Engineering, Johns Hopkins University, Baltimore, Maryland, USA. ${ }^{1}$ These authors contributed equally to this work.

*Corresponding author: seulki@jhmi.edu

Disclosure of Potential Conflicts of Interest: No potential conflicts of interest were disclosed.

Keyword: apoptosis, death receptors, nanomedicine, TRAIL, TRAIL sensitizers 


\section{Abstract}

Tumor necrosis factor-related apoptosis inducing ligand (TRAIL) has attracted great interest as a cancer therapy because it selectively induces death receptor (DR)-mediated apoptosis in cancer cells while sparing normal tissue. However, recombinant human TRAIL demonstrates limited therapeutic efficacy in clinical trials, possibly due to TRAIL-resistance of primary cancers and its inherent short half-life. Here we introduce drug delivery approaches to maximize in vivo potency of TRAIL in TRAIL-resistant tumor xenografts by (1) extending the half-life of the ligand with PEGylated TRAIL (TRAIL ${ }_{\mathrm{PEG}}$ ) and (2) concentrating a TRAIL sensitizer, selected from in vitro screening, in tumors via tumor-homing nanoparticles. Antitumor efficacy of TRAIL $_{\text {PEG }}$ with tumor-homing sensitizer was evaluated in HCT116 and HT-29 colon xenografts. Western blot, real-time PCR, immunohistochemistry and cell viability assays were employed to investigate mechanisms of action and antitumor efficacy of the combination. We discovered that doxorubicin (DOX) sensitizes TRAIL-resistant HT-29 colon cancer cells to TRAIL by upregulating mRNA expression of DR5 by $60 \%$ in vitro. Intravenously administered free DOX does not effectively upregulate DR5 in tumor tissues nor demonstrate synergy with TRAIL PEG $_{\text {in }}$ HT-29 xenografts, but rather introduces significant systemic toxicity. Alternatively, when DOX was encapsulated in hyaluronic acid-based nanoparticles (HAC/DOX) and intravenously administered with TRAIL ${ }_{\mathrm{PEG}}$, DR-mediated apoptosis was potentiated in HT-29 tumors by upregulating DR5 protein expression by $70 \%$ and initiating both extrinsic and intrinsic apoptotic pathways with reduced systemic toxicity compared to HAC/DOX or free DOX combined with TRAIL $_{\text {PEG }}(80 \%$ vs. $40 \%$ survival rate; $75 \%$ vs. $34 \%$ tumor growth inhibition). This study demonstrates a unique approach to overcome TRAIL-based therapy drawbacks using sequential administration of a tumor-homing TRAIL sensitizer and long-acting TRAILPEG. 


\section{Introduction}

Recombinant human tumor necrosis factor (TNF)-related apoptosis inducing ligand (rhTRAIL) and its agonistic antibodies have been under intense focus as crucial, molecularly targeted, antitumor biologics [1,2]. Unlike conventional anticancer agents and even other TNF family members, rhTRAIL selectively transduces apoptotic signals by binding to death receptors (DRs) that are widely expressed in most cancers, TRAIL-R1/DR4 and TRAIL-R2/DR5, while sparing normal cells [3-5]. This high tumor specificity along with broad applicability across multiple cancer types and proven safety in humans make TRAIL an ideal candidate for cancer therapy [68]. However, recent clinical trials of rhTRAIL, e.g. dulanermin, or humanized DR agonistic monoclonal antibodies, tested as either a monotherapy or combined with anticancer agents have failed to demonstrate benefits in cancer patients compared with historical controls [9-12]. The disappointing results raise concerns for the therapeutic implications of rhTRAIL. We identify two challenges that need to be overcome to adapt TRAIL-based agents as therapeutics - natural resistance and poor pharmacokinetics. We address these challenges using a drug delivery strategy with a targeted drug carrier and modified form of TRAIL.

The primary challenge to tackle in TRAIL-based therapy is natural resistance. The majority of primary cancer cells are TRAIL-resistant [11-13]. Mechanisms of TRAIL resistance are distinct among cancer cell types; however, they commonly comprise of: reduced cell surface DR expression, inhibited caspase-8 activation - the initiator caspase, up-regulated anti-apoptotic molecules such as Bcl-2 and the inhibitors of apoptosis (IAP) family proteins, and reduced expression of pro-apoptotic proteins like $\operatorname{Bax} / \operatorname{Bak}[14,15]$. The role of diverse molecules like anticancer agents and natural compounds in sensitizing TRAIL-resistant cancer cells has been investigated and introduced as an addition to TRAIL monotherapy. TRAIL-based combinations 
were well validated in vitro and in a few in vivo cancer models; however, they fail to demonstrate a similar synergy in cancer patients. Many reported examples utilize very high doses of chemotherapeutics for TRAIL sensitization that are limited for in vivo application in both dosing frequency and toxicity. This implies a need for alternative approaches to enable rhTRAIL combination therapy in the clinic. In this report, we utilize targeted drug carriers to achieve appropriate TRAIL sensitizer accumulation directly at the site of action.

In addition to TRAIL-resistance, rhTRAIL has an extremely short half-life in physiological conditions, 3-5 min in rodents and less than $30 \mathrm{~min}$ in humans $[16,17]$. It is widely accepted that wild-type proteins with short half-lives do not exhibit similar biological potency in physiological conditions as those tested in vitro [18]. Use of a more stable form of rhTRAIL with an extended half-life is expected to improve TRAIL action in physiological conditions, particularly for a biologic with an exceptionally short half-life like TRAIL. We firstly developed a series of long-acting PEGylated TRAILs (TRAIL ${ }_{\mathrm{PEG}}$ ) by PEGylating an isoleucine-zipperfused TRAIL (iLZ-TRAIL), a TRAIL variant that is known to be more potent than rhTRAIL [19]. PEGylation is considered the gold standard for half-life extension and a highly efficient commercial strategy as proven by PEGylated interferons and other FDA-approved biologics [20]. TRAIL $_{P E G}$ has increased stability over rhTRAIL with a significantly longer circulation half-life in rats $[21,22]$. As a result, TRAIL ${ }_{P E G}$ demonstrated superior in vivo anticancer potencies in xenografts bearing TRAIL-sensitive HCT116 colon cancer tumors over iLZ-TRAIL. Increasing the circulation time of TRAIL is still not a solution for targeting primary tumors associated with TRAIL resistance at the molecular level.

By integrating recent findings from basic and clinical studies in TRAIL biology and therapy, we hypothesize that TRAIL can have clinical efficacy in cancer by simultaneously 
addressing two key limitations, TRAIL resistance and its short half-life. First, we selected a TRAIL sensitizer in TRAIL-resistant colon cancer cells through cell-based screening and explored TRAIL and apoptotic signals at the molecular level. Next, the selected TRAIL sensitizer alone or formulated with tumor-homing polymer nanoparticles were systemically administered to xenografts bearing TRAIL-resistant tumors followed by TRAIL ${ }_{P E G}$ administration to investigate a synergistic effect on TRAIL-induced apoptosis in vivo. Lastly, we show the necessary conditions to potentiate anticancer efficacy of TRAIL with a select, tumorhoming TRAIL sensitizer and TRAIL variant in vivo. These studies demonstrate that strategies that address the short half-life of TRAIL alone or TRAIL resistance alone are not effective and hence may explain the disappointing clinical results of TRAIL-based cancer therapies thus far. Rather, a broad approach of addressing the two key TRAIL disadvantages can provide insight towards a viable clinical option for TRAIL-based therapies.

\section{Material and Methods}

\subsection{In Vitro Studies of TRAIL ${ }_{\mathrm{PEG}}$ Sensitivity in Human Cancer Cell Lines}

\subsubsection{Cell Culture}

HT-29, SW620, HCT116, and MDA-MB-231 cells were maintained in RPMI 1640 medium (Sigma, St. Louis, MO) supplemented with 10\% fetal bovine serum (FBS; Life Technology, Carlsbad, CA), $1 \%$ penicillin, and 1\% streptomycin (Life Technology). Cells were cultured at $37^{\circ} \mathrm{C}$ under an atmosphere of $5 \% \mathrm{CO}_{2}$. PC-3 and $\mathrm{A} 549$ cells were maintained in $\mathrm{F}-12 \mathrm{~K}$ medium (Sigma) supplemented with 10\% FBS, 1\% penicillin, and 1\% streptomycin. HEK293T cells were cultured in Modified Eagles Medium (MEM) (Sigma) supplemented with 10\% FBS, $1 \%$ penicillin, and 1\% streptomycin. These cell lines were purchased from ATCC (Manassas, VA). 
The cell lines were not authenticated was by the authors. Typically, $2 \times 10^{5}$ cells per well were plated in 6-well plates for treatment of agents.

\subsubsection{Cell Viability}

A total of $1 \times 10^{4}$ cells were plated in $0.1 \mathrm{~mL}$ in 96 -well flat bottom plates and incubated for $24 \mathrm{~h}$ before being exposed to various stimuli. After incubation for the indicated times, $5 \mu \mathrm{g} / \mathrm{mL}$ MTT solution was added to each well and incubated for $1 \mathrm{~h}$. After removal of the medium, $200 \mu \mathrm{L}$ of DMSO was added to each well to dissolve the formazan crystals. The absorbance at $540 \mathrm{~nm}$ was determined using a microplate reader (Bio-Tek Instruments, Inc, Winooski, VT). Triplicate wells were assayed for each condition.

\subsection{TRAIL Signaling and Apoptosis Analysis}

\subsubsection{In Situ DNA Strand Break Labeling (TUNEL assay)}

Tumor tissues were recovered from euthanized animals. Sections $(5 \mu \mathrm{m})$ were cut from $10 \%$ neutral buffered, formalin-fixed, paraffin- embedded tissue blocks. Apoptotic cell death in tumor tissues was visualized by performing TdT-mediated dUTP nick end labeling (TUNEL) assays according to the manufacturer instructions (Roche Mannheim, Germany).

\subsubsection{Antibodies and Western Blotting}

Anti-caspase-8 (Cell Signaling Technology, Danvers, MA, \#9746), anti-cleaved PARP-1 (Cell Signaling Technology, \#5625), anti-cleaved caspase-3 (Cell Signaling Technology, \#9664), anticleaved caspase-9 (Cell Signaling Technology, \#7237), anti-CD44 (Cell Signaling Technology, \#5640), anti-p-JNK (Cell Signaling Technology, \#4668), anti-p-p53 (Ser15 Cell Signaling Technology, \#9284), anti-BCl-2 (Cell Signaling Technology, \#2870), anti-p-BCL-2 (Cell Signaling Technology, \#2875), anti-BCL-XL (Cell Signaling Technology, \#2764), anti-DR4 (Abcam, Cambridge, MA, \#13890), anti-DR5 (Abcam, \#47179), anti-c-Jun (Santa Cruz 
Biotechnology, Santa Cruz, CA, sc-1694), or anti- $\beta$-actin (sc-47778) were used in Western blot analysis. In general, cells were lyzed and sonicated briefly in ice-cold PBS buffer (1 mM PMSF, and $1 \mu \mathrm{g} / \mathrm{ml}$ each of aprotinin, leupeptin, and pepstatin A). Cell lysates were clarified by centrifugation, resolved by SDS-PAGE, and proteins on gels were transferred to nitrocellulose (Bio-Rad, Hercules, CA) using a semidry blotter (Bio-Rad). The membrane was blocked with 3\% BSA in TBST (10 mM Tris-Cl, pH 8.0, $150 \mathrm{mM} \mathrm{NaCl}, 0.05 \%$ Tween-20) and incubated overnight at $4^{\circ} \mathrm{C}$ with primary antibodies. Immunoblots were visualized by an enhanced chemiluminescence method and analyzed by Multigauge software (Fujifilm, Tokyo, Japan).

\subsubsection{DR5 siRNA Transfection}

HT-29 cells were cultured in 6 well plates for $24 \mathrm{~h}$ and the cells were transfected with DR5 siRNA (Santa Cruz Biotechnology, Santa Cruz, CA, sc-40237) or control siRNA for $48 \mathrm{~h}$. Transfection was carried out using Lipofectamine 2000 reagent (Invitrogen) following the manufacturer's instructions.

\subsubsection{Quantitative RT (reverse transcription)-PCR}

Total cellular RNA was purified from HT-29 cells using Trizol reagent (Life Technology) and subjected to amplification with SuperScript One-Step RT-PCR system (Life Technology). Realtime PCR was carried out using a StepOne ${ }^{\mathrm{TM}}$ Real-Time PCR System according to the manufacturer instructions (Life Technology). The mean cycle threshold value (Ct) from triplicate samples was used to calculate the gene expression. $\beta$-actin was used as an internal control to normalize the variability in expression. Experiment was repeated three times with identical results. The following specific primers sets that are consensus region among isoforms were used for PCR; DR4, forward 5'-TGT GAC TTT GGT TGT TCC GTT GC-3' and reverse 5'-ACC TGA GCC GAT GCA ACA ACA G-3'; DR5, forward 5'- AAG ACC CTT GTG CTC GTT GT-3' and 
reverse 5'-AGG TGG ACA CAA TCC CTC TG-3'; actin, forward 5'- TCC CTG GAG AAG AGC TAC GA-3' and reverse 5'-AGC ACT GTG TTG GCG TAC AG-3'.

\subsubsection{Death-Inducing Signaling Complex Immunoprecipitation}

After HT-29 cells achieved $80 \%$ confluence, the cells were pretreated with doxorubicin for $24 \mathrm{~h}$ and then incubated with 500 ng/mL Flag-TRAIL (Enzo Life Sciences, Farmington, NY) for 30 min at $37^{\circ} \mathrm{C}$. The cells were lysed with DISC IP lysis buffer (30 mM Tris, $\mathrm{pH} \mathrm{7.4,} 150 \mathrm{mM} \mathrm{NaCl}$, $10 \%$ glycerol, $1 \%$ Triton $\mathrm{X}-100$ with $1 \mathrm{mM}$ PMSF, and $1 \mu \mathrm{g} / \mathrm{mL}$ each of aprotinin, leupeptin, and pepstatin A). Cell lysates were incubated with Flag (M2) beads (Sigma) overnight. The beads were subsequently washed three times with cold PBS, resolved onto SDS-PAGE gels and subjected to Western blot analysis.

\subsection{Analysis of TRAIL Sensitizer Cellular Uptake and Tumor Accumulation}

\subsubsection{Confocal Analysis}

HT-29 cells grown on coverslips in 12-well plates were treated with indicated agents. The cells were fixed in $4 \%$ paraformaldehyde for 5 min and then washed with ice-cold PBS (pH 8.0) three times. Finally, the cells were mounted on slides for visualization under a Fluoview FV10i-DOC confocal microscope (Olympus Optical, Tokyo, Japan).

\subsubsection{Flow Cytometry}

Cells were harvested, washed with PBS, re-suspended in $75 \%$ ethanol in PBS, and kept at $4^{\circ} \mathrm{C}$ for $30 \mathrm{~min}$. Cells were re-suspended with $1 \mathrm{mM}$ EDTA, $0.1 \%$ Triton-X-100 and $1 \mathrm{mg} / \mathrm{ml}$ RNAse A in PBS. The suspension was then analyzed on a FACSCaliber. The histogram in Fig. S4 was generated using the MultiCycle software (Phoenix Flow Systems, San Diego, CA, USA).

\subsubsection{DOX Distribution in HT-29 Xenograft Tumors}


Mice bearing HT-29 xenograft tumors were intravenously administered with DOX (7 mg/kg) and HAC/DOX (containing $7 \mathrm{mg} / \mathrm{kg}$ equivalent doxorubicin) when tumors reached $300 \mathrm{~mm}^{3}$. At each selected time point, 3 mice in one group were euthanized by cervical dislocation. Whole blood was collected via cardiac puncture with a heparinized syringe. Tumors were dissected out and frozen at $-70^{\circ} \mathrm{C}$ immediately. Plasma samples were isolated from whole blood by centrifugation at $3000 \mathrm{~g}$ for $5 \mathrm{~min}$. Tissues homogenates were prepared in $800 \mu \mathrm{L}$ water using a Polytron homogenizer (Brinkman Instruments, Mississauga, Ontario, Canada), and then $200 \mu \mathrm{L}$ of $\mathrm{H}_{2} \mathrm{SO}_{4}$ was added to the tissue homogenates. The solutions were then digested for $2 \mathrm{~h}$ at $60^{\circ} \mathrm{C}$. After the vials cooled to room temperature, $100 \mu \mathrm{L}$ of $\mathrm{AgNO}_{3}$ was added. Then the samples were centrifuged at 12,000g for $10 \mathrm{~min}$, and the supernatant was counted in a fluorospectrometer (RF5301, Shimadzu) at an excitation wavelength of $500 \mathrm{~nm}$ and emission wavelength of $558 \mathrm{~nm}$. The concentration of doxorubicin in each tissue was calculated based on a calibration curve. The calibration curve was linear over the 0.02 and $2.00 \mu \mathrm{g} / \mathrm{mL}$ range with a correlation coefficient of $\mathrm{R}^{2}=0.9993$.

\subsection{In Vivo Efficacy Studies of TRAIL $L_{P E G}$ in Xenograft Mice Models}

\subsubsection{HCT116 Xenograft Model}

All experiments involving tumor xenografts were performed according to protocols approved by the Johns Hopkins Animal Care and Use Committee and animal studies were undertaken in accordance with the rules and regulations. Freshly harvested HCT116 cells $\left(3 \times 10^{6}\right.$ cells/mouse)

were inoculated s.c. into BALB/c athymic mice $(\mathrm{n}=5)$. When tumor volume reached $\sim 50 \mathrm{~mm}^{3}$,

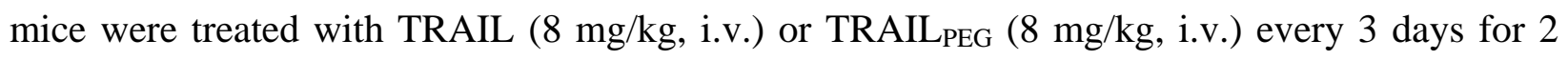
weeks (total 4 times). Tumor volumes were monitored for 30 days after tumor cell administration. Tumor volumes were calculated using longitudinal $(\mathrm{L})$ and transverse $(\mathrm{W})$ diameters using $\mathrm{V}=$ 
$\left(\mathrm{L}^{*} \mathrm{~W}^{2}\right) / 2$, and tumor growth inhibition (TGI) percent values were calculated using the formula TGI $\%=\left(1-\mathrm{TV}_{\text {sample }} / \mathrm{TV}_{\text {control }}\right) \times 100$, where $\mathrm{TV}$ is tumor volume

\subsubsection{HT-29 Xenograft Model}

The antitumor effects of TRAIL ${ }_{P E G}$ after HAC/DOX sensitizing were investigated in HT-29 tumor bearing mice $(n=5)$. Briefly, freshly harvested HT-29 cells $\left(5 \times 10^{6}\right.$ cells/mouse $)$ were inoculated s.c. into BALB/c athymic mice. Treatment was initiated when the tumors reached a mean volume of $150 \mathrm{~mm}^{3}$. Mice were treated with three rounds of DOX or HAC/DOX (7 mg/kg, i.v.) combined with $\mathrm{TRAIL}_{\mathrm{PEG}}(8 \mathrm{mg} / \mathrm{kg}$, i.v. $)$ for 10 days. The tumors were analyzed and calculated as described above $(\mathrm{n}=5)$.

\subsection{Statistical Analysis}

All data were analyzed by GraphPad Prism 6 (GraphPad Software, La Jolla, CA). Differences between two means were assessed by a paired or unpaired t-test. Differences among multiple means were assessed, as indicated, by one-way ANOVA, followed by Turkey's post-hoc test or by the Student's $t$-test as appropriate. Error bars represent S.D or S.E.M as indicated. $P$-values $<$ 0.05 were considered to be significant.

\section{Results}

3.1. TRAIL ${ }_{P E G}$ improves pharmacokinetics and reduces tumor growth in TRAIL-sensitive tumor xenografts but does not influence apoptosis in TRAIL-resistant tumors.

TRAIL $_{P E G}$ engineered with a $20 \mathrm{kDa}$ PEG molecule was synthesized as previously reported and used throughout the study. Earlier PK studies in rodents demonstrate 20kDa TRAILPEG has a half-life $\left(\mathrm{t}_{1 / 2}\right)$ of $12.3 \pm 2.2 \mathrm{~h}$ in mice (intraperitoneal injection), which is over 11-fold greater than free iLZ-TRAIL [22]. TRAIL $L_{P E G}$ also showed a 21 -fold increase in area under the curve 
(AUC) over TRAIL [22]. To compare pharmacodynamics (PD) between iLZ-TRAIL and

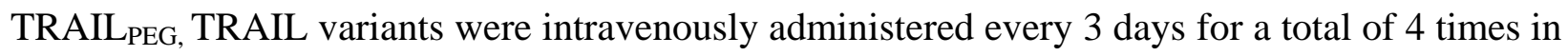
HCT116 xenografts when the tumor was palpable $\left(50 \mathrm{~mm}^{3}\right)$ (Fig. 1A). HCT116 is a human colon cancer cell line that is relatively sensitive to TRAIL-induced apoptosis. Compared to iLZTRAIL, TRAIL ${ }_{\mathrm{PEG}}(200 \mu \mathrm{g}$, protein-based) showed increased tumor growth inhibition (TGI) values (at day 28 for iLZ-TRAIL and TRAIL $\mathrm{PEG}_{2} ; 27 \%$ and 58\%, respectively). At the end of the study, tumor tissues were harvested and apoptotic cells in tumor sections were visualized by TdT-mediated dUTP nick and labeling (TUNEL) assay (Fig. 1B). TRAILPEG clearly showed tumor cell apoptosis in vivo compared to marginal signs in the iLZ-TRAIL-treated group. Next, we examined if the improved TRAIL stability of TRAIL ${ }_{\mathrm{PEG}}$ contributes to apoptosis in TRAILresistant tumors. A panel of known TRAIL-resistant human tumor cell lines including colon (HT-29, SW620), prostate (PC3), breast (MDA-MB-231R, MCF7) and lung (A549) as well as TRAIL-sensitive HCT116 and normal human kidney HEK293T cells were incubated with 1 $\mu \mathrm{g} / \mathrm{mL}$ of iLZ-TRAIL or TRAIL ${ }_{\mathrm{PEG}}$ for $3 \mathrm{~h}$ and $24 \mathrm{~h}$ in respective media. TRAIL sensitivities were expressed as induced cell death (\%), calculated as the percentage relative to the untreated

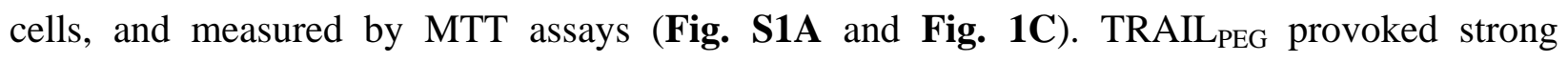
apoptosis only in TRAIL-sensitive HCT116 cells, like iLZ-TRAIL, as evidenced by cleavage of poly(ADP-ribose) polymerase 1 (PARP-1), a substrate of caspase-3 (Fig. S1B). This study

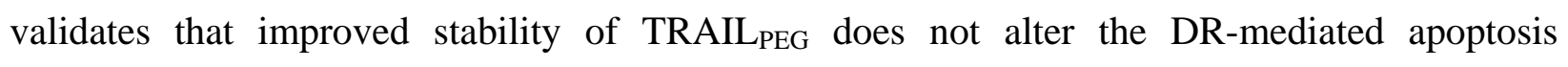
signaling in TRAIL-resistant tumors; thus, an additional strategy to extend the $t_{1 / 2}$ of TRAIL is needed to target both TRAIL-sensitive and -resistant tumors in vivo. 
A

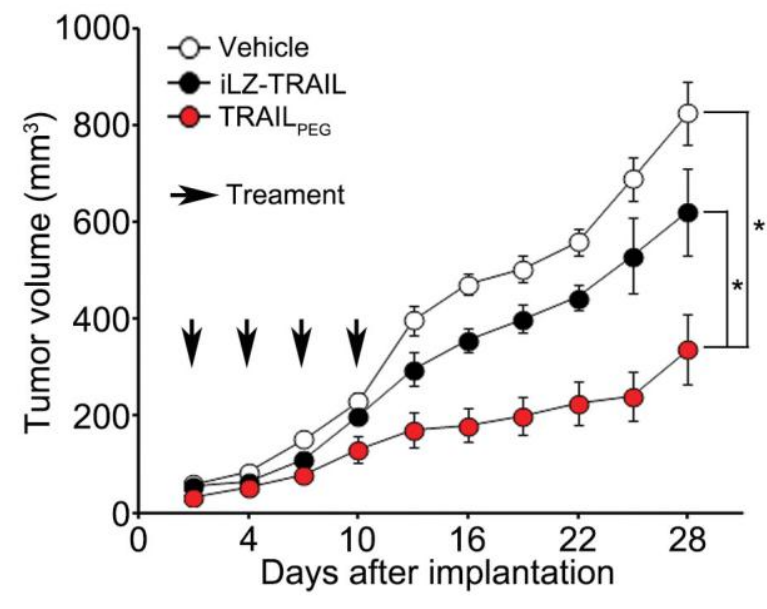

B

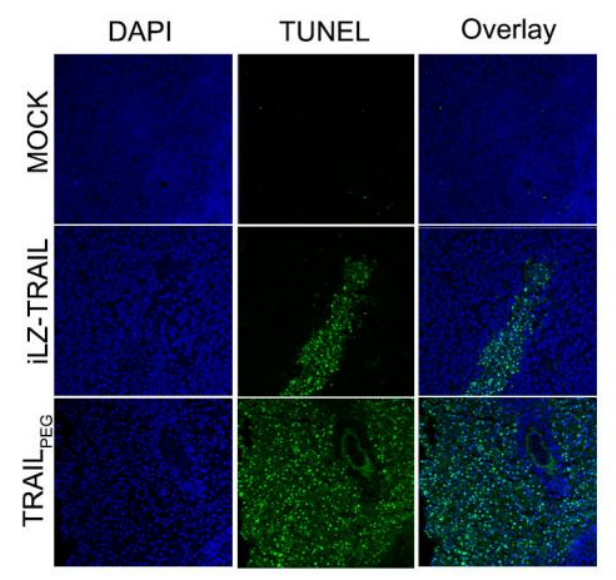

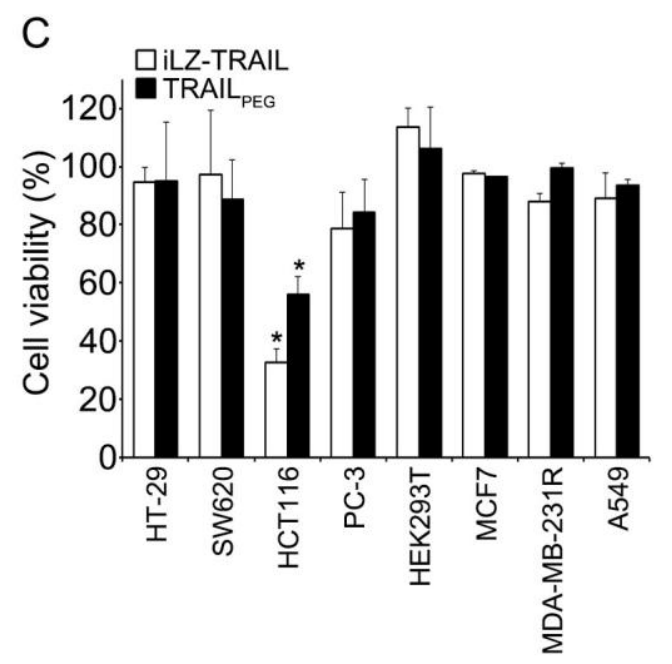

Figure 1. TRAIL $L_{P E G}$ has superior tumor growth inhibition effects over non-PEGylated TRAIL in TRAIL-sensitive xenografts, despite having a lower potency in vitro. (A) HCT116 xenografts were established and mice were intravenously treated when the tumor was palpable with four rounds of saline, iLZ-TRAIL (200 $\mu \mathrm{g})$ or TRAIL PEG $(200 \mu \mathrm{g}$, protein-based). Tumor volumes were determined by caliper measurements ( $\mathrm{n}=5$ /group). Values indicate means \pm SEM. (B)

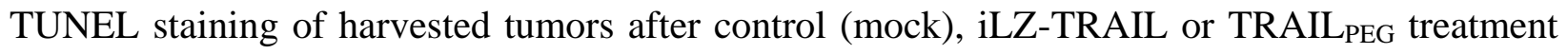
from (A). Fluorescence images were acquired under a confocal microscope and overlaid with Hoechst 33258 staining. (C) Human tumor cell lines: colon (HT-29, SW620, HCT116), prostate (PC3), breast (MDA-MB-231R, MCF7) and lung (A549) and normal human cell line: kidney (HEK293T) were collected and examined for their sensitivities to iLZ-TRAIL and TRAIL PEG $_{\text {by }}$ cell viability assay. Cells were treated with TRAIL variants $(1 \mu \mathrm{g} / \mathrm{mL}$, protein-based) for $24 \mathrm{~h}$ and cell death rates were measured by MTT assay $(\mathrm{n}=3)$. Values indicate means \pm SD. ${ }^{*} P<$ 0.001 vs. control group (without any treatment). 


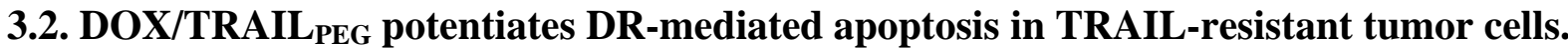

Accumulating reports suggest that various FDA-approved chemotherapies sensitize cancer cells to TRAIL-induced apoptosis. To identify synergism with TRAIL ${ }_{\mathrm{PEG}}$, common DNA damaging agents approved for colon cancer treatment, including doxorubicin (DOX), 5-fluorouracil (5-Fu), cisplatin (CIS), and irinotecan (IRINO), were incubated in TRAIL-resistant HT-29 cells with or without TRAIL ${ }_{\mathrm{PEG}}$ and screened for apoptosis. Lower doses of agents $(0.5 \mu \mathrm{g} / \mathrm{mL}$ of DOX, CIS; $1 \mu \mathrm{g} / \mathrm{mL}$ of $5-\mathrm{Fu}$ and $0.6 \mu \mathrm{g} / \mathrm{mL}$ of IRINO) were pretreated in HT-29 cells for $24 \mathrm{~h}$ followed by

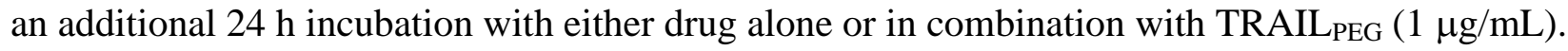
The low dose of drugs did not induce apoptosis based on the percentage of relative cell death (Fig. S1C). At high toxic doses (> $10 \mu \mathrm{g} / \mathrm{mL})$, most of the drug-treated cells were dead in $24 \mathrm{~h}$ (data not shown). When HT-29 cells were exposed to sublethal doses of DOX ( $2 \mu \mathrm{g} / \mathrm{mL}), 5$-FU (10 $\mu \mathrm{g} / \mathrm{mL})$, CIS $(2 \mu \mathrm{g} / \mathrm{mL})$, or IRINO ( $3 \mu \mathrm{g} / \mathrm{mL})$ combined with TRAIL ${ }_{\text {PEG }}$, enhanced TRAILinduced apoptosis was observed compared to drug alone (Fig. 2A). Among tested agents, DOX/TRAIL ${ }_{\mathrm{PEG}}$ combination clearly enhanced apoptosis through the proteolytic activation of caspase-8 (Casp-8) and caspase-9 (Casp-9) and consequently cleaved PARP-1 in HT-29 cells (Fig. 2B). Treatment of DOX also led to the phosphorylation of p53 and the activation of c-jun, a downstream substrate of c-Jun N-terminal kinase (JNK). Next, DOX/TRAIL PEG $_{\text {combination }}$ treatment was examined for enhanced apoptosis in different TRAIL-resistant cells. Individually, TRAIL $_{\text {PEG }}$ or DOX failed to induce PARP-1 cleavage in TRAIL-resistant human tumor cell lines, including HT-29, MDA-MB-231R, A549, and PC3. When combined, PARP-1 activation was significant in all TRAIL-resistant and TRAIL-sensitive cell lines examined, (Fig. 2C) and such synergism was exemplified cell death assays (Fig. S1D). To investigate if enhanced apoptosis by DOX/TRAIL ${ }_{\mathrm{PEG}}$ is DR-mediated through death-inducing signaling complex (DISC) formation, 
TRAIL DISC immunoprecipitation (IP) was assessed in HT-29 cells after treatment of DOX, TRAIL $_{\text {PEG }}$ or DOX/TRAIL ${ }_{\text {PEG }}$ followed by DR4 and DR5 Western blotting (Fig. 2D). Interestingly, TRAIL-induced DISC demonstrated the recruitment of DR5, but not DR4, on the cellular membrane after DOX/TRAIL treatment. To further validate the DR5 specific regulation, we used siRNA to determine DOX-mediated DR5 up-regulation. DR5 was highly induced by DOX treatment in HT-29 cells but did not upregulate with DR5 siRNA transfection followed by DOX treatment (Fig. 2E). As examined by quantitative real-time PCR (qPCR), DOX increased DR5 mRNA by $60 \%$ in HT-29 cells compared to untreated cells, whereas DR4 mRNA levels did not change (Fig. 2F).

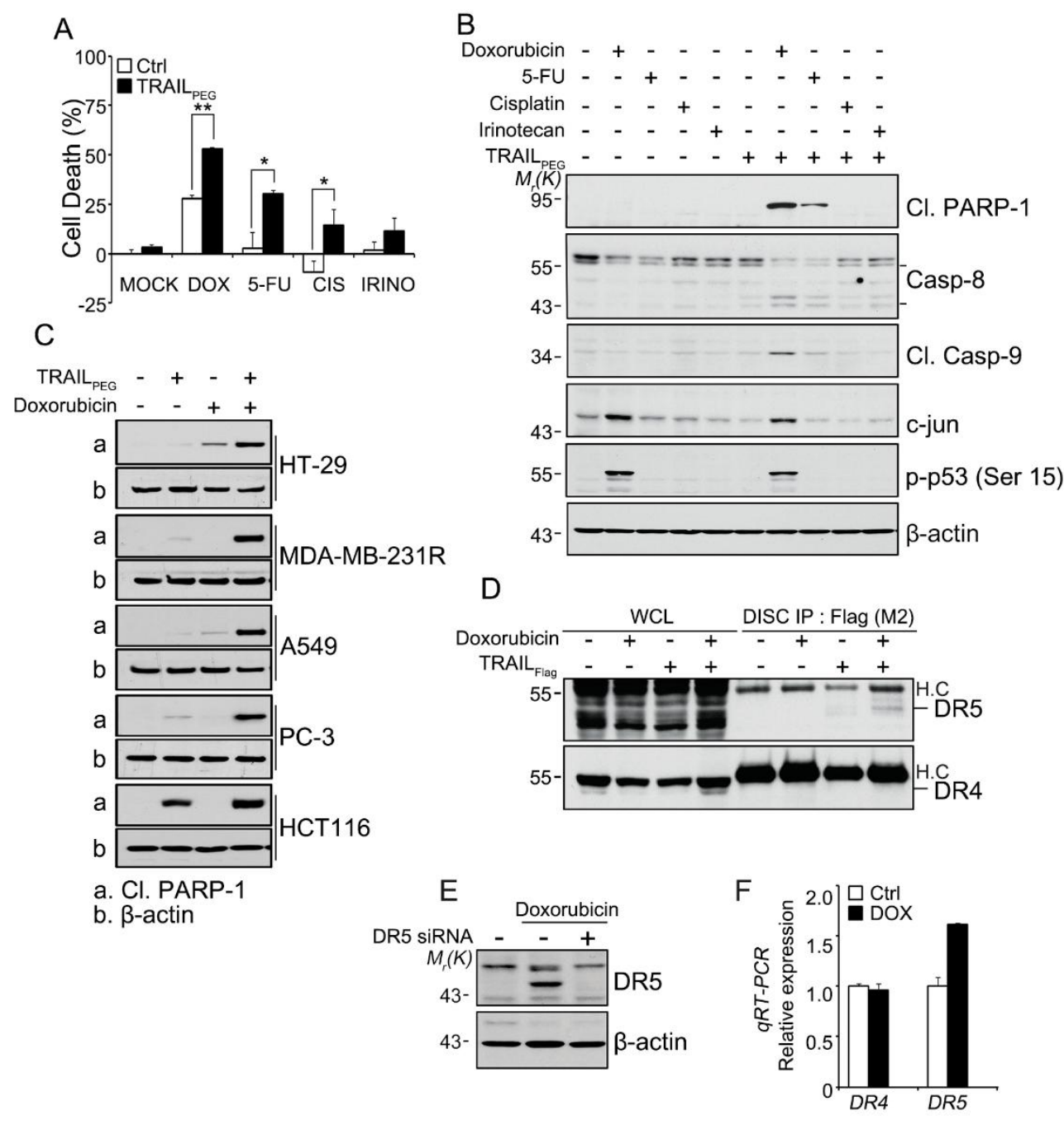


Figure 2. Doxorubicin (DOX) induces apoptosis when combined with TRAIL ${ }_{\mathrm{PEG}}$ in TRAILresistant cancer cell lines. (A) DNA damaging agents sensitize TRAIL-resistant HT-29 cells to cell death after TRAILPEG treatment. HT-29 cells were treated with sublethal doses of doxorubicin (DOX, $2 \mu \mathrm{g} / \mathrm{mL}$ ), 5-fluorouracil (5-FU, $10 \mu \mathrm{g} / \mathrm{mL}$ ), cisplatin (CIS, $2 \mu \mathrm{g} / \mathrm{mL}$ ) and irinotecan (IRINO, $2.9 \mu \mathrm{g} / \mathrm{mL}$ ) for $24 \mathrm{~h}$ and further incubated with TRAIL PEG $(1 \mu \mathrm{g} / \mathrm{mL}$ ) for an additional $24 \mathrm{~h}$. The cell death rates were measured by MTT assay $(\mathrm{n}=3)$. ${ }^{*} P<0.001$ vs. cells treated with cytotoxic agent only $(\mathrm{Ctrl})$. Values indicate means \pm SD. (B) The cell extracts were prepared and the levels of apoptosis-related proteins were examined by western blotting; cleaved PARP-1 (Cl. PARP-1), caspase-8 (Casp-8), cleaved Casp-8, c-Jun and phospho-p53 (p-p53). $\beta$ -

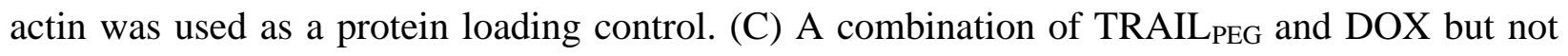
drug alone sensitizes TRAIL-induced apoptosis as seen by cleaved PARP-1, a hallmark of apoptosis, in various TRAIL-resistant cells, HT-29 (colon), MDA-MB-231 (breast), A549 (lung), and PC3 (prostate), as in TRAIL-sensitive HCT116 (colon) cancer cells. (D) DISC formation in HT-29 cells. HT-29 cells were left untreated or stimulated with $500 \mathrm{ng} / \mathrm{ml}$ of TRAILFlag for $1 \mathrm{~h}$. The lysates were immunoprecipitated with FLAG (M2) and analyzed by Western blotting using DR4 and DR5 antibodies. WCL: Whole cell lysates. (E and F) DR5 induction in HT-29 cells by DOX. (E) HT-29 cells were transfected with DR5 siRNA for $48 \mathrm{~h}$ and the cells were left untreated or incubated with DOX for an additional $24 \mathrm{~h}$. Cell extracts were examined by western blotting for DR5 using anti-DR5 and anti- $\beta$-actin antibodies. $\beta$-actin was used as a protein loading control. (F) HT-29 cells were treated with DOX for $24 \mathrm{~h}$ and the cell extracts were examined for mRNA levels of DR4 and DR5 using gene-specific primers by qRT-PCR analysis.

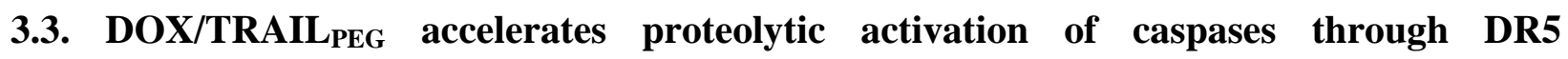

upregulation in HT-29 cells. It has been reported that HT-29 cells are TRAIL-resistant because of low DR5 expression on the cellular membrane $[23,24]$. In other reports, DOX has been demonstrated to sensitize TRAIL-induced apoptosis by affecting the cell surface localization of DR5 in colon cancer cells [25]. To explore how DOX and DOX/TRAIL ${ }_{\mathrm{PEG}}$ enhance apoptosis, HT-29 cells were treated with DOX or DOX/TRAIL ${ }_{\text {PEG }}$ at different time points. When HT-29 cells were pre-sensitized with DOX $(2 \mu \mathrm{g} / \mathrm{mL})$ and then treated with TRAIL ${ }_{\text {PEG }}$ for $24 \mathrm{~h}$, Casp-8 and Casp-3 activated (Fig. 3A and 3B). Regardless of TRAILPEG, DOX upregulated DR5 expression (3 to 4-fold), but not DR4, at the protein level. TRAIL intrinsically binds to both DR4 and DR5, but we have shown that only altered protein expression of DR5 in HT-29 cells plays a 
critical role in TRAIL-induced apoptosis while DR4 levels remain unchanged. To assess if the enhanced DOX/TRAIL ${ }_{P_{E G}}$-induced apoptosis is due to altered DR5 expression, we synthesized a peptide-based dimeric DR5 antagonist (DR5-A) based on a reported sequence of YCKVILTHRCY [26] (Fig. S2 and Fig. S3A). The neutralizing efficacy of DR5-A was confirmed by treating HCT116 cells with DR5-A $(5,10 \mu \mathrm{g} / \mathrm{mL})$ and TRAIL ${ }_{\text {PEG }}$ or DR5 agonistic antibody (Fig. S3B and Fig S3C). Upon incubation, the DR5-A effectively blocked TRAIL ${ }_{\mathrm{PEG}}$-induced apoptosis by neutralizing DR5 as evidenced by the reduced cleavage of Casp-3 and PARP-1. With this antagonistic peptide, we investigated the extent of DR5 expression induced by DOX treatment and its effect on DOX/TRAIL ${ }_{\mathrm{PEG}}$-induced apoptosis in HT-29 cells. When TRAIL ${ }_{\text {PEG }}$ was co-treated with both DOX and DR5-A to HT-29 cells, cell death evoked by the DOX/TRAIL ${ }_{\mathrm{PEG}}$ treatment was significantly inhibited by $70 \%$ compared to that of cells without DR5-A treatment (Fig. 3C). Blocking DR5 substantially decreased the proteolytic activation of Casp-8, Capse-9 and PARP-1 cleavage in cells treated with DOX/TRAIL ${ }_{\text {PEG }}$ while showing no effect on BCL2/BCL-XL expression that was mainly reduced by DOX (Fig. 3E). It has been reported that JNK mediates DOX- or TRAIL-induced apoptosis in cancer cells [27]. In addition, activation of the JNK pathway leads to DR upregulation in multiple tumor cells including colon cancer [28, 29]. To study this, HT-29 cells were treated with DOX and TRAIL ${ }_{P E G}$ alone or in combination with SP600125 $(20 \mu \mathrm{M})$, a JNK inhibitor [30]. Consequently, inhibition of JNK phosphorylation reduced DOX and TRAILPEG-induced cell death by 35\% (Fig. 3D) and suppressed proteolytic activation of Casp-8, Casp-9 and PARP-1 cleavages (Fig. 3F). However, SP600125 had no effect on regulating DR5, indicating DOXinduced DR5 upregulation is not stimulated by the JNK pathway. This suggests that JNK 
partially mediates DOX/TRAIL ${ }_{\mathrm{PEG}}$-induced apoptosis but is not involved in DR5 upregulation in HT-29 cells.
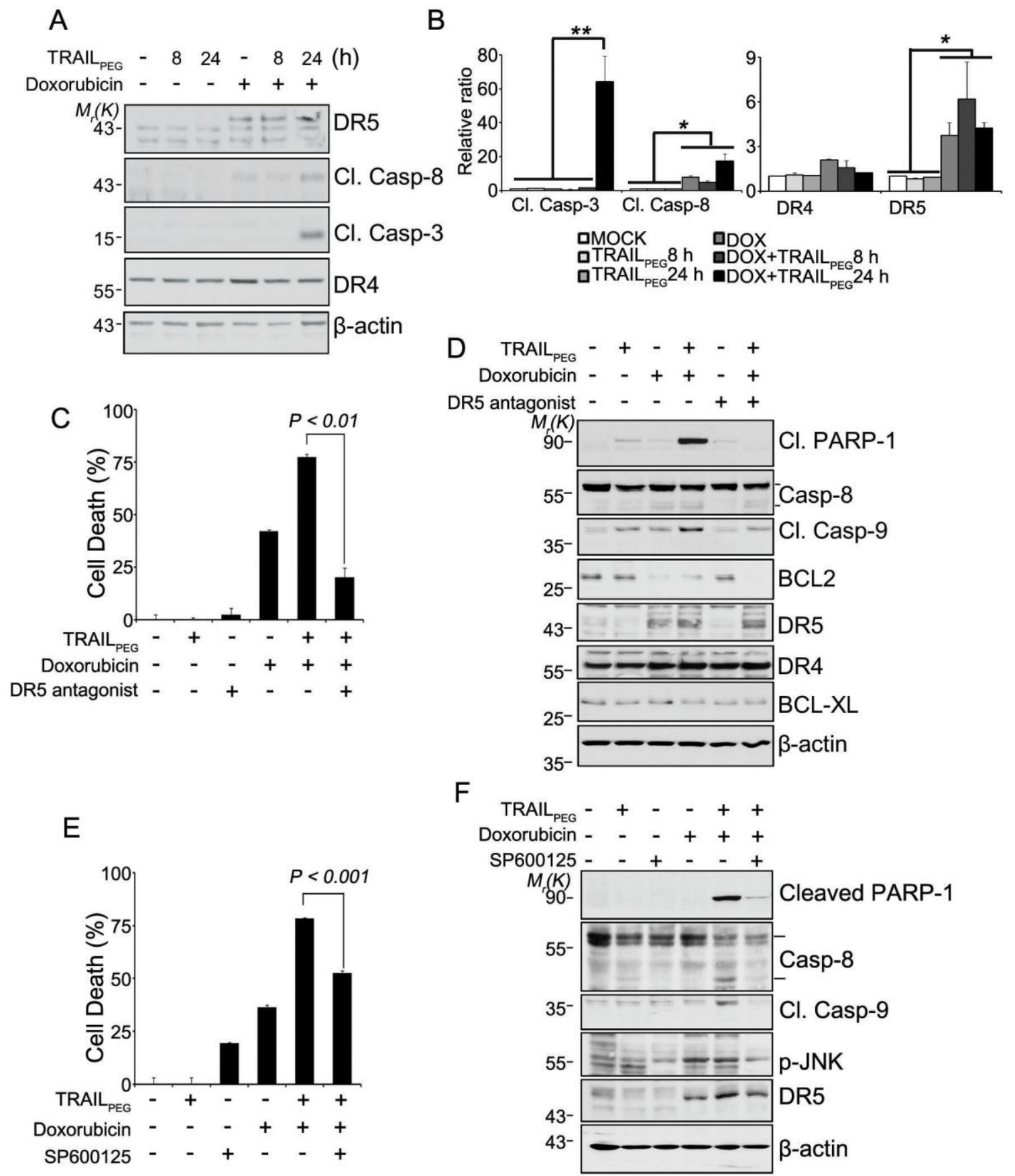

Figure 3. When combined with TRAIL ${ }_{P E G}$, DOX synergizes TRAIL-induced apoptosis in HT-29 cells through DR5 upregulation and partially by JNK-mediated apoptosis. (A) Western blotting

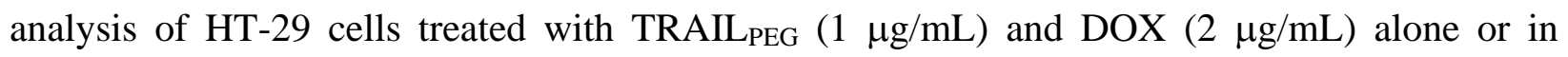


combination with different incubation times. The cell extracts were prepared and the levels of DR4, DR5 and cleaved caspase-8 (Cl. Casp-8) and caspase-3 (Cl. Casp-3) were examined. (B) The relative fold increase of cleaved caspase-3, caspase-8, DR4 and DR5 expressions from control group (no TRAIL ${ }_{\mathrm{PEG}}(1 \mu \mathrm{g} / \mathrm{mL})$ and DOX $(2 \mu \mathrm{g} / \mathrm{mL})$ treatment). $* P<0.05, * * P<0.001$ vs. groups (C) The effect of upregulated DR5 on TRAIL-induced cell death in HT-29 cells. Cells were treated with DOX $(2 \mu \mathrm{g} / \mathrm{mL})$ and TRAIL PEG $(1 \mu \mathrm{g} / \mathrm{mL})$ alone or in combination with or without DR5-A ( $2 \mu \mathrm{g} / \mathrm{mL}$, DR5 antagonist peptide) pretreatment. Cell death rates were measured by MTT assay $(\mathrm{n}=3) . * P<0.001$ vs. DR5 neutralized group. (D) Western blotting analysis of cells as treated in (C). Cleaved caspases, PARP-1, DR4 and DR5, BCL2, BCL-XL and $\beta$-actin (loading control) was measured. (E) The effect of JNK on TRAIL-induced cell death in HT-29 cells. Cells were treated with DOX $(2 \mu \mathrm{g} / \mathrm{mL})$ and TRAIL $\operatorname{PEG}(1 \mu \mathrm{g} / \mathrm{mL})$ alone or in combination with or without SP600125 (JNK inhibitor, $20 \mu \mathrm{M}$ ) pre-treatment. Cell death rates were measured by MTT assay $(\mathrm{n}=3)$. ${ }^{*} P<0.001$ vs. JNK activity inhibited group. (F) Cleaved caspases, PARP-1, phosphorylated JNK and DR5 western blot analysis of cells in (E). $\beta$-actin was measured as a loading control.

\subsection{Tumor-homing HAC/DOX but not free DOX accumulates DOX concentration in}

tumor tissues in vivo. In many cases, select anticancer agents acting as TRAIL sensitizers in vitro were not fully validated in animal models and when in vivo efficacy was demonstrated, relatively high doses of drugs were needed to effectively sensitize TRAIL-resistant tumors in vivo. However, such exceedingly high doses of chemotherapeutic agents as TRAIL sensitizers are not clinically practical. One effective way to utilize such toxic agents as a sensitizer while minimizing systemic toxicity in vivo is using a tumor-homing drug delivery system. We previously optimized a hyaluronic acid-based conjugate (HAC), a tumor-homing nanocarrier system comprised of biocompatible hyaluronic acid, that can deliver small molecules to the intracellular space of cancer cells via CD44 receptors with reduced systemic toxicity [31, 32]. Importantly, this targeted, intracellular delivery was observed and verified in different in vivo cancer models, ranging from colon and melanoma to head and neck [32-37]. In aqueous

solutions, the HAC structure can self-assemble into a nanocarrier sequestering the 
hydrophobic/amphiphilic molecules to the center of the particle. Because of HAC's abundant functional groups, the surface of HAC can be modified with fluorophores or other detectable moieties for tracking and imaging in cells and in vivo [35]. The schematic diagram and chemical structure of HAC is shown in Fig. 4A. CD44 expression and therefore HAC drug delivery is dependent on cell types. Among the tested cells, HT-29, HCT116, MDA-MB-213R and A549 tumor cells express CD44 whereas SW620 and HEK293T cells do not express high levels of CD44 (Fig. 4B). DOX is well-encapsulated within HAC (HAC/DOX) with a high loading content (21\%,wt) and loading efficiency (71\%) having a mean diameter of $206 \mathrm{~nm}$ in PBS (10 mM, pH 7.4) (Table S1 and Fig. S4A). When DOX was incorporated in HAC labeled with fluorescein molecules and treated to HT-29 cells, HAC/DOX showed rapid cellular uptake after 10 min of incubation and saturated at $1 \mathrm{~h}$ (Fig. 4C and Fig. S4B). Importantly, HAC/DOX promptly burst releases the incorporated DOX inside the cell, which is supported by the restored quenched fluorescence of DOX based on microscopy images (Fig. S4C). HAC was shown to be non-toxic in our previous studies $[32,36]$. The tumor-homing delivery of DOX by HAC was studied in tumor xenograft models. DOX concentration in plasma and tumor tissues was quantified by fluorescence absorbance followed by an extraction process [38]. When HAC/DOX and the same dose of DOX dissolved in saline was intravenously injected in HT-29 xenografts bearing approximately $300 \mathrm{~mm}^{3}$ tumors, HAC/DOX delivered more DOX in the harvested tumor tissues than free DOX. The concentration of free DOX in the tumor region gradually decreased with time at $6 \mathrm{~h}$ post drug administration (Fig. 4D). In contrast, HAC/DOX markedly increased DOX accumulation in the tumor region from $6 \mathrm{~h}$ to $24 \mathrm{~h}$ and maintained accumulation $48 \mathrm{~h}$ postinjection. HAC/DOX demonstrated 12-fold and 55-fold increased accumulation of DOX in isolated tumors at $24 \mathrm{~h}$ and $48 \mathrm{~h}$, respectively, compared to that of DOX alone. To confirm DOX 
distribution in tumors, harvested tumor sections isolated at $48 \mathrm{~h}$ were stained with DAPI and visualized by confocal microscopy (Fig. 4E and Fig. S5A). As expected, HAC/DOX treated tissues showed greater DOX accumulation compared to control.

A
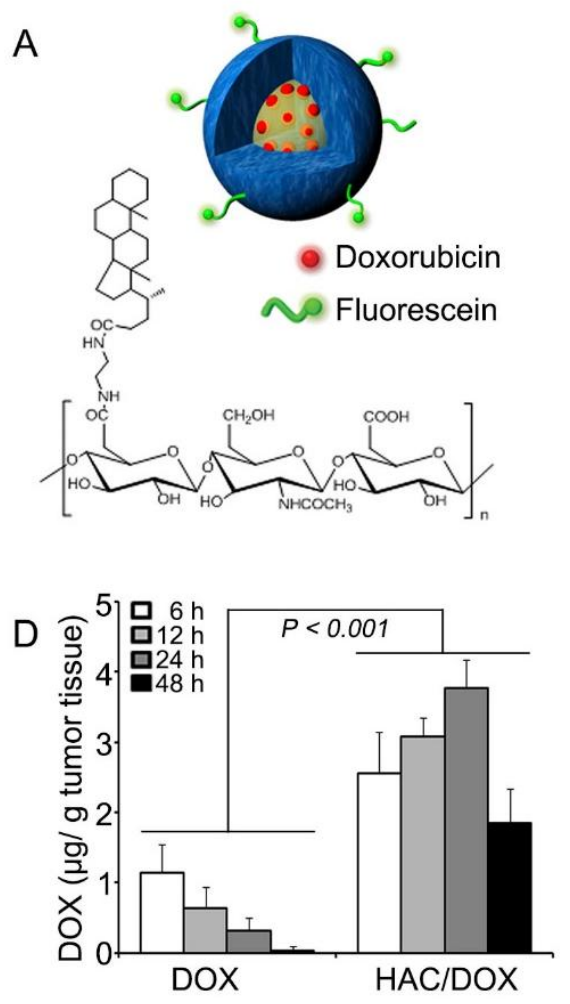

B
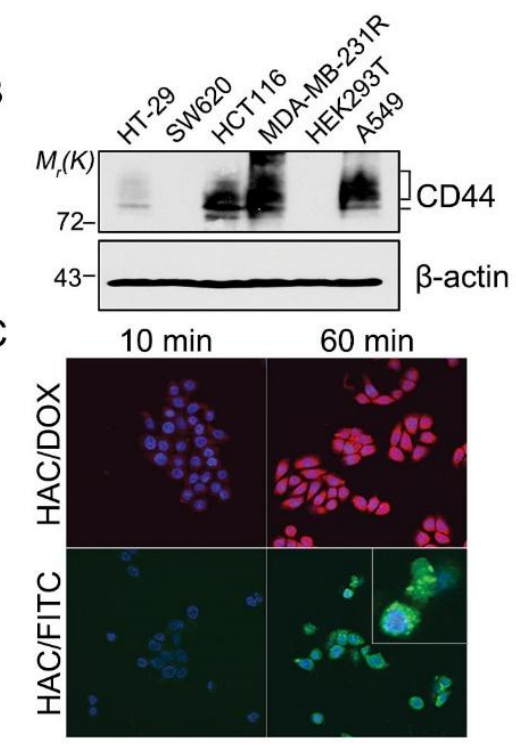

$E$

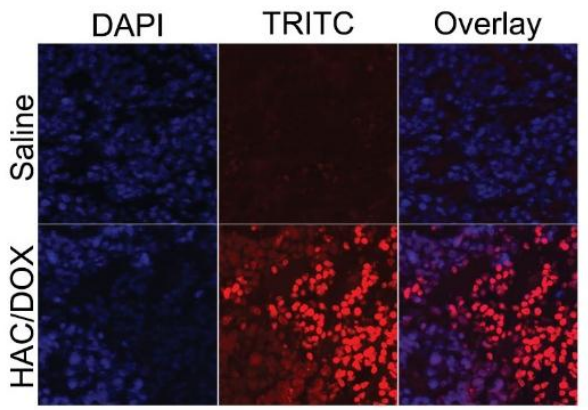

Figure 4. HAC/DOX but not free DOX accumulates in tumors for a sustained period of time and potentiates caspase cascade when combined with TRAIL PEG. $_{\text {. }}$ (A) Upper; schematic diagram of HAC/DOX, hyaluronic acid-based conjugate (HAC) carrying doxorubicin in the core and FITC dye molecules labeled on the surface for fluorescence microscopy. Lower; a chemical structure of HAC, hyaluronic acid chemically conjugated with cholanic acid. (B) Cancer cells were examined for their CD44 expression. The cell extracts were prepared and the levels of CD44 were examined by western blotting. (C) HAC/DOX rapidly internalizes and releases DOX in HT-29 cells. HT-29 cells were incubated with HAC/DOX ( $2 \mu \mathrm{g} / \mathrm{mL}$, doxorubicin-based $)$ for 10 min and $60 \mathrm{~min}$. Fluorescence images were acquired under a confocal microscope and overlaid with DAPI staining. Representative images are based on four different measurements. HAC; green (FITC, ex/em: 490/525), DOX; red (TRITC, ex/em: 557/576), and nucleus; blue (DAPI, 
ex/em: 350/470). FACS analysis described in Supplementary Fig. S4. (D) DOX concentration in the harvested tumors following single intravenous dosing of DOX (7 mg/kg) and HAC/DOX (7 $\mathrm{mg} / \mathrm{kg}$, DOX-based) in HT-29 xenografts. When tumors reached a diameter of $300 \mathrm{~mm}^{3}$, mice were intravenously treated with DOX and HAC/DOX. At the indicated time points, mice were sacrificed and the tumor concentration of doxorubicin were measured by a fluorescence absorbance method followed by extraction recovery $(n=3)$. Values indicate means \pm S.D. (E) The uptake and distribution of doxorubicin in tumor tissues. Representative fluorescence images of tumor sections demonstrate the high accumulation of doxorubicin after HAC/DOX injection. Nucleus; blue (DAPI ex/em: 350/470), doxorubicin; red (TRITC; ex/em: 557/576).

\subsection{A tumor-homing HAC/DOX combined with long-acting TRAIL PEG potentiates} apoptosis in TRAIL-resistant tumor xenografts with reduced systemic toxicity. After confirming that HAC/DOX predominantly accumulates in tumors in vivo, the next study

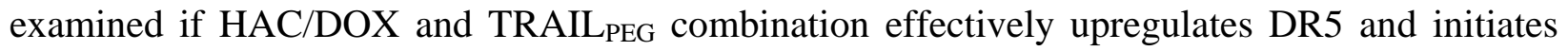
apoptosis in vivo as demonstrated in vitro. When tumor volumes reached $200 \mathrm{~mm}^{3}$, HT-29 xenografts were intravenously treated with TRAILPEG, HAC/DOX and HAC/DOX/TRAIL PEG $(n=3)$. Because a caspase cascade was potentiated in HT-29 cells only when DOX was pretreated

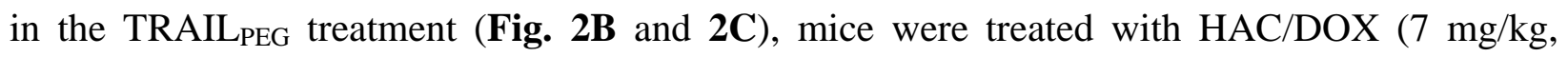
DOX-based) $24 \mathrm{~h}$ before TRAIL PEG $_{\text {treatment. After } 24 \mathrm{~h} \text { of TRAIL }}$ PEG treatment, the expression of DR5 and DR4 as well as Casp-8 and Casp-3 were analyzed in harvested tumor tissues. In accordance with cellular studies, HAC/DOX treatment increased the protein expression of DR5 in tumors by $70 \%$ in vivo while DR4 levels remained unchanged (Fig. 5A and 5B). In particular, neither HAC/DOX nor TRAIL ${ }_{\mathrm{PEG}}$ alone failed to initiate a caspase cascade. Casp-8 and Casp-3

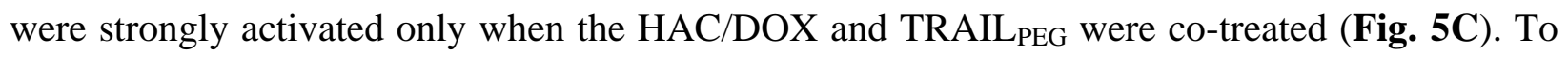
find a dose range in mice models, two TRAIL ${ }_{\mathrm{PEG}}$ formulations with different DOX concentrations, low (2 mg/kg, DOX-based, Dox $\left.{ }_{\text {low }}\right)$ and high $(7 \mathrm{mg} / \mathrm{kg}$, close to maximum tolerated dose, Dox high$_{\text {}}$, were injected in HT-29 xenografts when tumor volumes reached 200 $\mathrm{mm}^{3}$ followed by TRAIL $\mathrm{PEG}_{\mathrm{P}}$ treatment. Each tumor was harvested and analyzed by 
immunoblotting after $24 \mathrm{~h}$ of TRAIL ${ }_{P E G}$ treatment (Fig. S5B). After a single treatment, DOX at the low dose marginally increased the cleaved Casp- 9 and Casp- 8 but showed some enhanced activation at the high DOX dose. Interestingly, neither low nor high DOX doses alone altered cleaved Casp-3 levels, an indicator of apoptosis. In contrast, HAC/DOX combined with TRAIL $_{\text {PEG }}$ clearly initiated the caspase cascade by regulating cleaved Casp- 9 and Casp- 8 at both low and high DOX doses. HAC/DOX with low and high DOX concentrations significantly enhanced Casp-3 activation compared to DOX alone (for $\mathrm{DOX}_{\text {low }}$, DOX $\mathrm{Digh}_{\text {, }}$ HAC/DOX $\mathrm{low}_{\text {, }}$

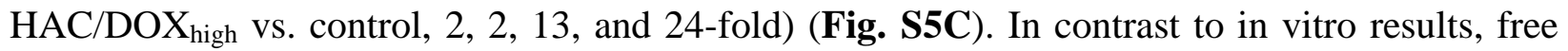
DOX at the high dose was shown to marginally alter cleavage of initiator caspases, Casp- 8 and Casp-9, and not executional caspase, Casp-3, when combined with TRAIL ${ }_{P E G}$ in vivo.

A

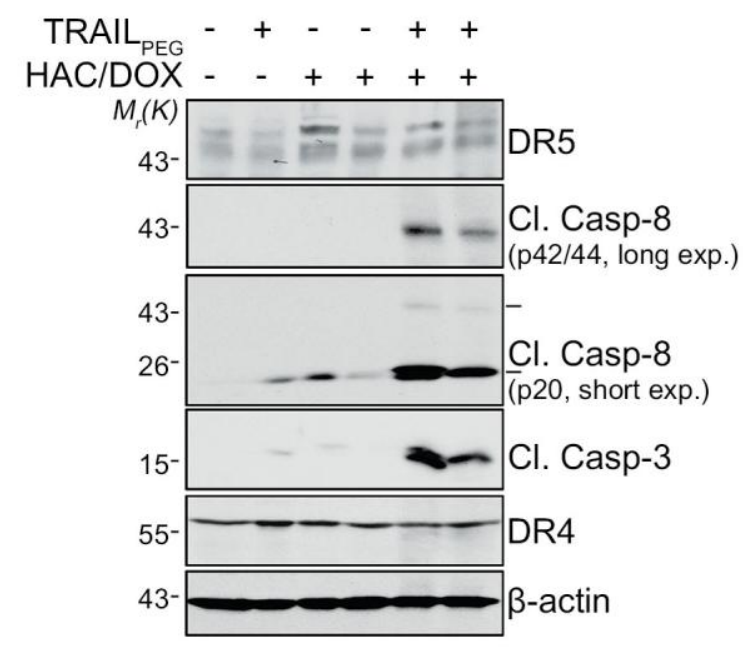

B
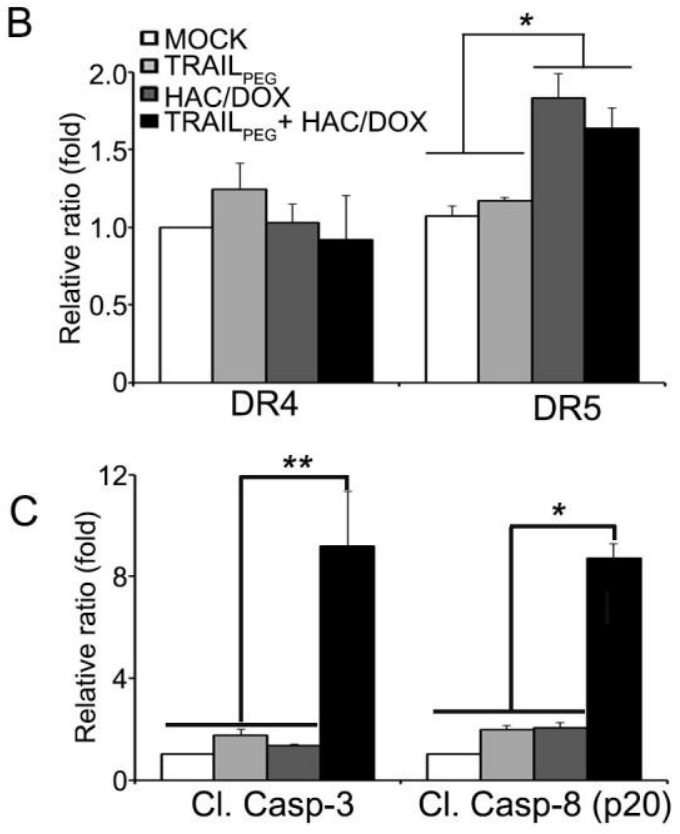

Figure 5. Simultaneous treatment of TRAIL ${ }_{\mathrm{PEG}}$ and HAC/DOX initiates apoptosis in TRAILresistant tumors in vivo. (A) Lysates of HT-29 tumors from mice treated with TRAIL PEG $_{2}(200 \mu \mathrm{g}$ per mouse) and HAC/DOX (7 mg/kg, DOX-based) alone or in combination were western blotted for death receptors (DR5, DR4), cleaved caspases and $\beta$-actin (loading control) expression 
analysis. Two representative western blot analyses are shown for each TRAILPEG group. (B, C) The relative fold increase of DR and caspase expressions $* P<0.05, * * P<0.001$ vs. groups.

After confirming the necessary condition to generate TRAIL-induced apoptosis in

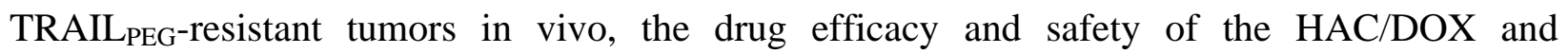
TRAIL $_{\text {PEG }}$ combination in HT-29 xenografts was examined. rhTRAIL was excluded from the

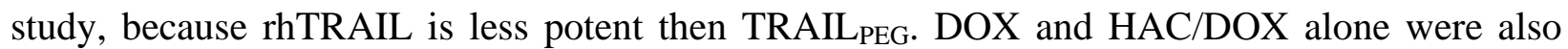
ruled out for the in vivo studies, because they do not efficiently induce apoptosis in HT-29 tumor models as presented earlier (Fig. 5). When tumor volumes reached $150 \mathrm{~mm}^{3}$, mice were intravenously treated with TRAILPEG alone or with DOX and HAC/DOX at a $7 \mathrm{mg} / \mathrm{kg} \mathrm{DOX}$ dose every 3 days for a total of 3 times as indicated in Fig. 6A. TRAIL ${ }_{P E G}$ alone marginally altered tumor growth just as was demonstrated by in vitro studies. In contrast, the two TRAIL PEG combinations suppressed tumor growth. TGI values were significantly decreased by the HAC/DOX and TRAIL ${ }_{P E G}$ combination throughout the study period (at day 28, for TRAIL ${ }_{\text {PEG, }}$ DOX and TRAIL PEG $_{\text {HAC/DOX and TRAIL }}$ PEG; 14, 34, and 75\% respectively). It should be noted that $60 \%$ of mice treated with a high dose of free DOX died during the treatment cycle due to the toxicity of the agent (Fig. 6B). Although carrying the same amount of high dose, HAC/DOX demonstrated a significantly improved tolerability in terms of survival rate, allowing the use of a necessary high dose of TRAIL sensitizer in vivo. 
A

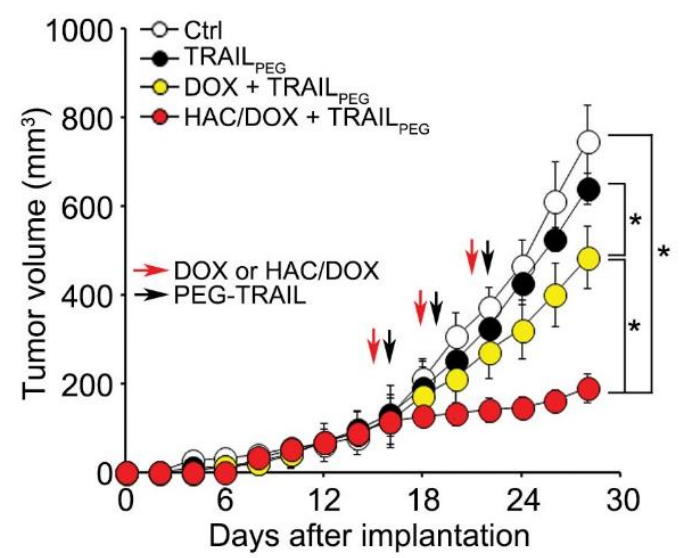

B

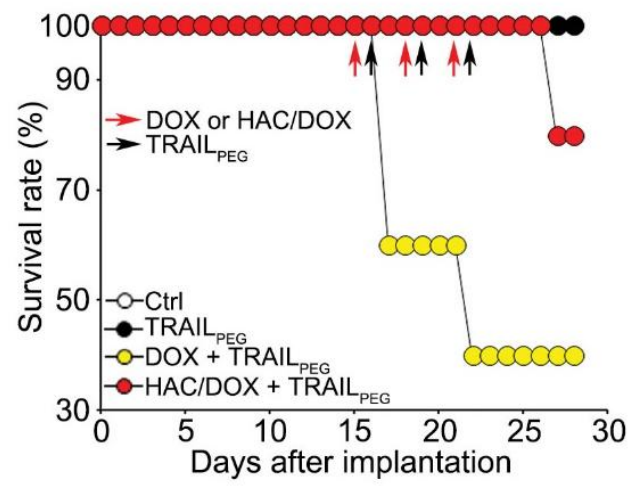

Figure 6. Simultaneous treatment of TRAIL ${ }_{P E G}$ and HAC/DOX reduces tumor growth in TRAIL-resistant tumors and reduces toxicity by DOX in vivo. (A) Mice bearing approximately $150 \mathrm{~mm}^{3}$ HT-29 tumors were intravenously treated with vehicle, TRAIL ${ }_{\text {PEG }}(200 \mu \mathrm{g})$ alone, TRAIL $_{\text {PEG }}(200 \mu \mathrm{g})$ combined with DOX ( $\left.7 \mathrm{mg} / \mathrm{kg}\right)$ or HAC/DOX (7 mg/kg, DOX-based) every 3 days starting at day 15 for a total of 3 doses. Tumor volumes were determined by caliper measurements ( $n=5 /$ group).Values indicate means \pm SEM. (B) Survival rate curve of mice treated in (A).

\section{Discussion}

TRAIL has unique features as a nontoxic anticancer therapy because it selectively induces apoptosis in a wide range of cancers while leaving normal cells unharmed. However, TRAILbased therapies tested in cancer patients, even when co-treated with anticancer agents, show marginal responses, suggesting a gap in the therapeutic approach between preclinical models and humans [9, 12]. To date, the critical reasons for such disappointing results are not clearly explained in the literature; and, more importantly, there are no clear alternatives to restore such TRAIL-based therapies in the clinic. Based on our studies, we conclude that an effective TRAIL therapy requires: 1) identification of the tumor's TRAIL sensitivity, 2) physiologically stable and active TRAIL-based compound, and 3) tumor-homing drug delivery techniques for TRAIL sensitizers. 
One important property usually neglected in the TRAIL research field is its inherent instability in physiological conditions. During the last few years, we put emphasis on the inherent short half-life of rhTRAIL and developed TRAIL ${ }_{P E G}$ analogs with an extended half-life. A stable isoleucine zipper (iLZ)-TRAIL is known to be more potent in vitro and in vivo compared to that of rhTRAIL because of the improved trimeric protein stability induced by the iLZ motif [39]. Additionally, TRAIL ${ }_{P E G}$ offers improved stability and solubility over rhTRAIL and iLZ fusion TRAIL as well as reduced aggregation at high concentrations, which enables easy handling of the protein in solution [21]. Trimeric TRAIL ${ }_{P E G}$ also has advantages over TRAIL agonistic antibodies in terms of safety and activity induced by clustering of DRs to induce DISC formation efficiently. Because TRAIL $_{\mathrm{PEG}}$ contains the native-type protein structure and PEGylation reduces immunogenicity of the compound, it is expected to be less immunogenic compared to humanized antibodies. TRAIL signaling is a complicated process associated with binding to multiple receptors, inciting reports of TRAIL's therapeutic potential in disorders other than cancer particularly in inflammatory diseases. Such roles could not be fully realized by utilizing DR antibodies that affect only a few receptors at most. From this point of view,

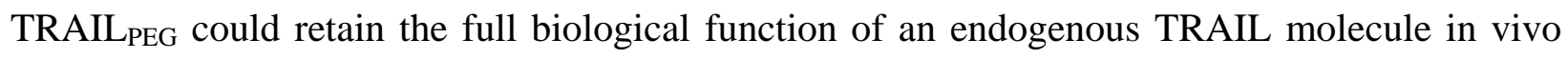
with a longer half-life. As a result, the full therapeutic potential of TRAIL can be investigated. Therefore, TRAIL ${ }_{\text {PEG }}$ shows greater in vivo anticancer activity than iLZ-TRAIL (Fig. 1A, B), despite having a lower potency in vitro (Fig. 1C). Nonetheless, TRAIL PEG $_{\text {showed marginal }}$ efficacy, similar to rhTRAIL, in TRAIL-resistant tumors examined in this study; thus, the results indicate a need for an additional strategy to address-TRAIL-resistance in vivo.

Diverse anticancer agents have been utilized as TRAIL sensitizers. In addition to their sensitizing roles in TRAIL-resistant tumors, a combinatory TRAIL and anticancer agent would 
be an ideal regimen for cancer therapy. TRAIL predominantly mediates an extrinsic pathway for apoptosis via the activation of caspase- 8 at the DISC. To a lesser degree, TRAIL also mediates the intrinsic pathway of apoptosis that results in the activation of caspase-9 through the release of cytochrome $\mathrm{c}$ from the mitochondria and the activation of other pro-apoptotic factors. Because most conventional anticancer drugs induce apoptosis through the intrinsic pathway, the interplay between the extrinsic and intrinsic apoptotic pathways would be an effective rationale for cancer therapy. In reality, however, dulanermin combined with anticancer drugs did not elicit synergistic effects in cancer patients [12]. We hypothesize that the accumulation of the anticancer drug in the tumor was too low to efficiently sensitize TRAIL-resistant tumors in patients. In many cases, high doses of TRAIL sensitizers were used in animal models of TRAILresistant tumors to induce a similar effect demonstrated in vitro. Such high dosing profiles are not applicable in the clinic, because they induce serious systemic toxicity. To overcome the limitation, we firstly screened the TRAIL ${ }_{\mathrm{PEG}}$ sensitizing efficacy of four widely used anticancer drugs at sublethal doses in TRAIL-resistant HT-29 colon cancer cells (Fig. 2). Based on the relative cell death and levels of cleaved PARP-1, a hallmark of apoptosis, the combination of DOX and TRAIL $L_{\text {PEG }}$ was selected for further studies. We validated that DOX enhances TRAIL sensitivity in HT-29 cells by upregulating DR5 specifically on the cellular membrane and, when combined with TRAIL ${ }_{P E G}$, increases Casp-3 cleavage (Fig. 3). Drug delivery by nanoparticles can reduce high dose DOX-induced systemic toxicity over free DOX by preferentially delivering its cargo to tumor tissues. Therefore, we utilized HAC/DOX as a tumor-homing TRAIL sensitizer to deliver DOX to tumor tissues and in turn sensitize tumor cells to TRAIL in vivo (Fig. 4A). HAC is a model platform for delivering cytotoxic agents, because HAC selectively targets tumors through two distinct mechanisms: passively accumulating in tumors through the 
enhanced permeability and retention (EPR) effect [40] followed by active targeting of CD44, a natural ligand of hyaluronic acid and an antigen overexpressed on various tumors [41]. In vivo, HAC/DOX prolonged DOX activity in the blood and accumulated DOX at higher concentrations in HT-29 tumor tissues after intravenous injection compared to DOX alone (Fig. 4B-E). Tumorbearing mice were sacrificed after $24 \mathrm{~h}$ of drug treatment, and tumor tissues were analyzed for TRAIL sensitization and apoptosis at the molecular level (Fig. 5). Importantly, intravenously administered free DOX at the high dose did not increase Casp-3 activity when combined with TRAIL $_{\text {PEG, }}$ whereas HAC/DOX clearly initiated the caspase cascade and potentiated TRAILinduced apoptosis. In particular, both cleaved Casp-8 and Casp-3 were apparent in tumor tissues only when xenografts were simultaneously treated with HAC/DOX and TRAIL PEG $_{\text {. Free DOX }}$ combined with TRAIL ${ }_{P E G}$ did not efficiently inhibit tumor growth but rather induced significant systemic toxicity (Fig. 6B). In accordance with in vitro and ex vivo study results, HAC/DOX combined with TRAIL ${ }_{P E G}$ clearly inhibited tumor growth in vivo with improved drug tolerability (Fig. 6).

TRAIL's proven safety and inherent cancer-selectivity warrants its potential as an ideal anticancer therapy, especially with reduced adverse effects compared to conventional first-line chemotherapy. The present study introduces one strategy to restore rhTRAIL-based therapy in the clinic by addressing at least two key limitations from a drug delivery approach; (1) utilizing rhTRAIL with an extended half-life and (2) effectively sensitizing TRAIL-resistant tumors through tumor-homing TRAIL sensitizers. The strategy can be tailored to include diverse TRAIL sensitizers, reported or novel, formulated with other types of tumor-homing nanoparticles, including nanoparticles in the clinical pipeline $[42,43]$. It remains to be investigated if the proposed strategy improves therapeutic efficacy in xenografts bearing different TRAIL-resistant 
tumor cells and metastatic tumors as well as in patient-derived xenograft models. Based on the high unmet clinical need for effective, less toxic anticancer therapies combined with the unique features of TRAIL in safety and applicability towards many cancer types, we anticipate that new TRAIL-based therapies could benefit cancer patients in the clinic. In our studies, we address two key limitations to first-generation TRAIL-based therapies in order to pave a path towards more promising clinical trials.

\section{Conclusion}

TRAIL's proven safety and inherent cancer-selectivity retains its potential as an ideal anticancer therapy, despite sobering clinical trials of recombinant TRAIL and more recent TRAIL receptor agonists. By integrating recent findings from clinical and basic science studies, this paper introduces a unique approach to initiate DR-mediated apoptosis in TRAIL-resistant tumors by simultaneously utilizing (1) a long-acting PEGylated iLZ-TRAIL to overcome the short half-life of TRAIL and (2) tumor-homing nanoparticles carrying chemotherapeutic agents as a TRAIL sensitizer to enable efficient TRAIL sensitization of tumors with low systemic toxicity. Our drug delivery strategy may warrant the resurgence of TRAIL-based therapies for clinical translation.

\section{Acknowledgments}

This work was supported by the US National Institutes of Health grants R00EB013450 (S.L) and the US Department of Defense grants, CA130460 (S.L., Y.O.) and PC131920 (M.S). This work also supported in part by the National Research Foundation of Korea grants, Global Research 
Laboratory Program NRF-2013K1A1A2002050115 (M.G.P., S.L., K.K. J.H.P), NRF2013R1A1A2062043 (K.C.L.) and NRF-2013R1A1A2064165 (S.M.L.). 


\section{References}

[1] A. Ashkenazi, R.S. Herbst, To kill a tumor cell: the potential of proapoptotic receptor agonists, J. Clin. Invest. 118 (2008) 1979-1990.

[2] R.W. Johnstone, A.J. Frew, M.J. Smyth, The TRAIL apoptotic pathway in cancer onset, progression and therapy. Nat. Rev. Cancer 8 (2008) 782-798.

[3] J.E. Allen, W.S. El-Deiry, Regulation of the human TRAIL gene. Cancer Biol. Ther. 13 (2012) 1143-1151.

[4] A. Ashkenazi, R.C. Pai, S. Fong, S. Leung, D.A. Lawrence, S.A. Marsters, C. Blackie, L. Chang, A. E. McMurtrey, A. Hebert, L. DeForge, I.L. Koumenis, D. Lewis, L. Harris, J. Bussiere, H. Koeppen, Z. Shahrokh, R.H. Schwall, Safety and antitumor activity of recombinant soluble Apo2 ligand. J. Clin. Invest. 104 (1999) 155-162.

[5] H. Walczak, R.E. Miller, K. Ariail, B. Gliniak, T.S. Griffith, M. Kubin, W. Chin, J. Jones, A. Woodward, T. Le, C. Smith, P. Smolak, R.G. Goodwin, C.T. Rauch, J.C. Schuh, D.H. Lynch, Tumoricidal activity of tumor necrosis factor-related apoptosis-inducing ligand in vivo, Nature Med. 5 (1999) 157-163.

[6] D.W. Stuckey, K. Shah, TRAIL on trial: preclinical advances in cancer therapy, Trends Mol. Med. 19 (2013) 685-694.

[7] R.S. Herbst, S.G. Eckhardt, R. Kurzrock, S. Ebbinghaus, P.J. O'Dwyer, M.S. Gordon, W. Novotny, M.A. Goldwasser, T.M. Tohnya, B.L. Lum, A. Ashkenazi, A.M. Jubb, D.S. Mendelson, Phase I dose-escalation study of recombinant human Apo2L/TRAIL, a dual proapoptotic receptor agonist, in patients with advanced cancer, J. Clin. Oncol. 28 (2010) 2839-2846.

[8] S. Leong, R.B. Cohen, D.L. Gustafson, C.J. Langer, D.R. Camidge, K. Padavic, L. Gore, M. Smith, L.Q. Chow, M. von Mehren, C. O'Bryant, S. Hariharan, S. Diab, N.L. Fox, R. 
Miceli, S.G. Eckhardt, Mapatumumab, an antibody targeting TRAIL-R1, in combination with paclitaxel and carboplatin in patients with advanced solid malignancies: results of a phase I and pharmacokinetic study. J. Clin. Oncol. 27 (2009):4413-4421.

[9] J.C. Soria, Z. Mark, P. Zatloukal, B. Szima, I. Albert, E. Juhasz, J.L. Pujol, J. Kozielski, N. Baker, D. Smethurst, Y.J. Hei, A. Ashkenazi, H. Stern, L. Amler, Y. Pan, F. Blackhall, Randomized phase II study of dulanermin in combination with paclitaxel, carboplatin, and bevacizumab in advanced non-small-cell lung cancer. J. Clin. Oncol. 29 (2011) $4442-$ 4451.

[10] T. Trarbach, M. Moehler, V. Heinemann, C.H. Kohne, M. Przyborek, C. Schulz, V. Sneller, G. Gallant, S. Kanzler, Phase II trial of mapatumumab, a fully human agonistic monoclonal antibody that targets and activates the tumour necrosis factor apoptosisinducing ligand receptor-1 (TRAIL-R1), in patients with refractory colorectal cancer. Br. J. Cancer 102 (2010) 506-512.

[11] L.Y. Dimberg, C.K. Anderson, R. Camidge, K. Behbakht, A. Thorburn, H.L. Ford, On the TRAIL to successful cancer therapy? Predicting and counteracting resistance against TRAIL-based therapeutics. Oncogene 32 (2013) 1341-1350.

[12] J. Lemke, S. von Karstedt, J. Zinngrebe, H. Walczak, Getting TRAIL back on track for cancer therapy. Cell Death Differ. 21 (2014) 1350-1364.

[13] T. Newsom-Davis, S. Prieske, H. Walczak, Is TRAIL the holy grail of cancer therapy? Apoptosis 14 (2009) 607-623.

[14] C.T. Hellwig, M. Rehm, TRAIL signaling and synergy mechanisms used in TRAILbased combination therapies. Mol. Cancer Ther. 11 (2012) 3-13. 
[15] C. Voelkel-Johnson, TRAIL-mediated signaling in prostate, bladder and renal cancer. Nat. Rev. Urol. 8 (2011) 417-427.

[16] S.K. Kelley, L.A. Harris, D. Xie, L. Deforge, K. Totpal, J. Bussiere, J.A. Fox, Preclinical studies to predict the disposition of Apo2L/tumor necrosis factor-related apoptosisinducing ligand in humans: characterization of in vivo efficacy, pharmacokinetics, and safety. J. Pharmacol. Exp. Ther. 299 (2001) 31-38.

[17] A. Ashkenazi, P. Holland, S.G. Eckhardt, Ligand-based targeting of apoptosis in cancer: the potential of recombinant human apoptosis ligand 2/Tumor necrosis factor-related apoptosis-inducing ligand (rhApo2L/TRAIL). J. Clin. Oncol. 26 (2008) 3621-3630.

[18] J.M. Harris, R.B. Chess, Effect of pegylation on pharmaceuticals. Nat. Rev. Drug Discov. 2(2003) 214-221.

[19] T.M. Ganten, R. Koschny, J. Sykora, H. Schulze-Bergkamen, P. Buchler, T.L. Haas, M.B. Schader, A. Untergasser, W. Stremmel, H. Walczak, Preclinical differentiation between apparently safe and potentially hepatotoxic applications of TRAIL either alone or in combination with chemotherapeutic drugs. Clin. Cancer Res. 12 (2006) 2640-2646.

[20] G. Pasut, F.M. Veronese, PEG conjugates in clinical development or use as anticancer agents: an overview. Adv. Drug Deliv. Rev. 61 (2009) 1177-1188.

[21] S.Y. Chae, T.H. Kim, K. Park, C.H. Jin, S. Son, S. Lee, Y.S. Youn, K. Kim, D.G. Jo, I.C. Kwon, X. Chen, K.C. Lee, Improved antitumor activity and tumor targeting of NH(2)terminal-specific PEGylated tumor necrosis factor-related apoptosis-inducing ligand. Mol. Cancer Ther. 9 (2010) 1719-1729. 
[22] T.H. Kim, Y.S. Youn, H.H. Jiang, S. Lee, X. Chen, K.C. Lee, PEGylated TNF-related apoptosis-inducing ligand (TRAIL) analogues: pharmacokinetics and antitumor effects. Bioconjug. Chem. 22 (2011) 1631-1637.

[23] K.G. Drosopoulos, M.L. Roberts, L. Cermak, T. Sasazuki, S. Shirasawa, L. Andera, A. Pintzas, Transformation by oncogenic RAS sensitizes human colon cells to TRAILinduced apoptosis by up-regulating death receptor 4 and death receptor 5 through a MEK-dependent pathway. J. Biol. Chem. 280 (2005) 22856-22867.

[24] Stolfi, R. Caruso, E. Franze, A. Rizzo, A. Rotondi, I. Monteleone, M.C. Fantini, F. Pallone, G. Monteleone, 2-methoxy-5-amino-N-hydroxybenzamide sensitizes colon cancer cells to TRAIL-induced apoptosis by regulating death receptor 5 and survivin expression. Mol. Cancer Ther. 10 (2011) 1969-1981.

[25] S. Lacour, O. Micheau, A. Hammann, V. Drouineaud, J. Tschopp, E. Solary, M.T. Dimanche-Boitrel, Chemotherapy enhances TNF-related apoptosis-inducing ligand DISC assembly in HT29 human colon cancer cells. Oncogene 22 (2003) 1807-1816.

[26] J. Vrielink, M.S. Heins, R. Setroikromo, E. Szegezdi, M.M. Mullally, A. Samali, W.J. Quax, Synthetic constrained peptide selectively binds and antagonizes death receptor 5. FEBS J. 277 (2010) 1653-1665.

[27] F. Muhlenbeck, E. Haas, R. Schwenzer, G. Schubert, M. Grell, C. Smith, P. Scheurich, H. Wajant, TRAIL/Apo2L activates c-Jun NH2-terminal kinase (JNK) via caspasedependent and caspase-independent pathways. J. Biol. Chem. 273 (1998) 33091-33098.

[28] N.G. Dolloff, P.A. Mayes, L.S. Hart, D.T. Dicker, R. Humphreys, W.S. El-Deiry, Offtarget lapatinib activity sensitizes colon cancer cells through TRAIL death receptor upregulation. Sci. Transl. Med. 3 (2011) 86ra50. 
[29] L. Fu, Y.D. Lin, H.A. Elrod, P. Yue, Y. Oh, B. Li, H. Tao, G.Z. Chen, D.M. Shin, F.R. Khuri, S.Y. Sun, c-Jun NH2-terminal kinase-dependent upregulation of DR5 mediates cooperative induction of apoptosis by perifosine and TRAIL. Mol. Cancer 9 (2010) 315.

[30] B.L. Bennett, D.T. Sasaki, B.W. Murray, E.C. O'Leary, S.T. Sakata, W. Xu, J.C. Leisten, A. Motiwala, S. Pierce, Y. Satoh, S.S. Bhagwat, A.M. Manning, D.W. Anderson, SP600125, an anthrapyrazolone inhibitor of Jun N-terminal kinase. Proc. Natl. Acad. Sci. U. S. A. 98 (2001) 13681-13686.

[31] K.Y. Choi, E.J. Jeon, H.Y. Yoon, B.S. Lee, J.H. Na, K.H. Min, S.Y. Kim, S.J. Myung, S. Lee, X. Chen, I.C. Kwon, K. Choi, S.Y. Jeong, K. Kim, J.H. Park, Theranostic nanoparticles based on PEGylated hyaluronic acid for the diagnosis, therapy and monitoring of colon cancer. Biomaterials 33 (2012) 6186-6193.

[32] K.Y. Choi, H. Chung, K.H. Min, H.Y. Yoon, K. Kim, J.H. Park, I.C. Kwon, S.Y. Jeong, Self-assembled hyaluronic acid nanoparticles for active tumor targeting. Biomaterials 31 (2010) 106-114.

[33] K.Y. Choi, H.Y. Yoon, J.H. Kim, S.M. Bae, R.W. Park, Y.M. Kang, I.S. Kim, I.C. Kwon, K. Choi, S.Y. Jeong, K. Kim, J.H. Park, Smart nanocarrier based on PEGylated hyaluronic acid for cancer therapy. ACS Nano 5 (2011) 8591-8599.

[34] K.Y. Choi, G. Saravanakumar, J.H. Park, K. Park, Hyaluronic acid-based nanocarriers for intracellular targeting: interfacial interactions with proteins in cancer. Colloids Surf. B Biointerfaces 99 (2012) 82-94.

[35] M. Swierczewska, K.Y. Choi, E.L. Mertz, X. Huang, F. Zhang, L. Zhu, H.Y. Yoon, J.H. Park, A. Bhirde, S. Lee, X. Chen, A facile, one-step nanocarbon functionalization for biomedical applications. Nano Lett. 12 (2012) 3613-3620. 
[36] G. Liu, K.Y. Choi, A. Bhirde, M. Swierczewska, J. Yin, S.W. Lee, J.H. Park, J.I. Hong, J. Xie, G. Niu, D.O. Kiesewetter, S. Lee, X. Chen, Sticky nanoparticles: a platform for siRNA delivery by a bis(zinc(II) dipicolylamine)-functionalized, self-assembled nanoconjugate. Angew. Chem. Int. Ed. Engl. 51 (2012) 445-449.

[37] H.Y. Yoon, H.R. Kim, G. Saravanakumar, R. Heo, S.Y. Chae, W. Um, K. Kim, I.C. Kwon, J.Y. Lee, D.S. Lee, J.C. Park, J.H. Park, Bioreducible hyaluronic acid conjugates as siRNA carrier for tumor targeting. J. Control. Release 172 (2013) 653-661.

[38] A. Rahman, D. Carmichael, M. Harris, J.K. Roh, Comparative pharmacokinetics of free doxorubicin and doxorubicin entrapped in cardiolipin liposomes. Cancer Res. 46 (1986) 2295-2299.

[39] J. Jiang, X. Liu, L. Deng, P. Zhang, G. Wang, S. Wang, H. Liu, Y. Su, GMP production and characterization of leucine zipper-tagged tumor necrosis factor-related apoptosisinducing ligand (LZ-TRAIL) for phase I clinical trial. Eur. J. Pharmacol. 740 (2014) $722-$ 732.

[40] H. Maeda, H. Nakamura, J. Fang, The EPR effect for macromolecular drug delivery to solid tumors: Improvement of tumor uptake, lowering of systemic toxicity, and distinct tumor imaging in vivo. Adv. Drug Deliv. Rev. 65 (2013) 71-79.

[41] S.C. Ghosh, S. Neslihan Alpay, J. Klostergaard, CD44: a validated target for improved delivery of cancer therapeutics. Expert Opin. Ther. Targets 16 (2012) 635-650.

[42] J. Hrkach, D. Von Hoff, M. Mukkaram Ali, E. Andrianova, J. Auer, T. Campbell, D. De Witt, M. Figa, M. Figueiredo, A. Horhota, S. Low, K. McDonnell, E. Peeke, B. Retnarajan, A. Sabnis, E. Schnipper, J.J. Song, Y.H. Song, J. Summa, D. Tompsett, G. Troiano, T. Van Geen Hoven, J. Wright, P. LoRusso, P.W. Kantoff, N.H. Bander, C. 
Sweeney, O.C. Farokhzad, R. Langer, S. Zale, Preclinical development and clinical translation of a PSMA-targeted docetaxel nanoparticle with a differentiated pharmacological profile. Sci. Transl. Med. 4 (2012) 128ra139.

[43] C.A. Schutz, L. Juillerat-Jeanneret, H. Mueller, I. Lynch, M. Riediker, Therapeutic nanoparticles in clinics and under clinical evaluation. Nanomedicine (Lond) 8 (2013) 449-467. 

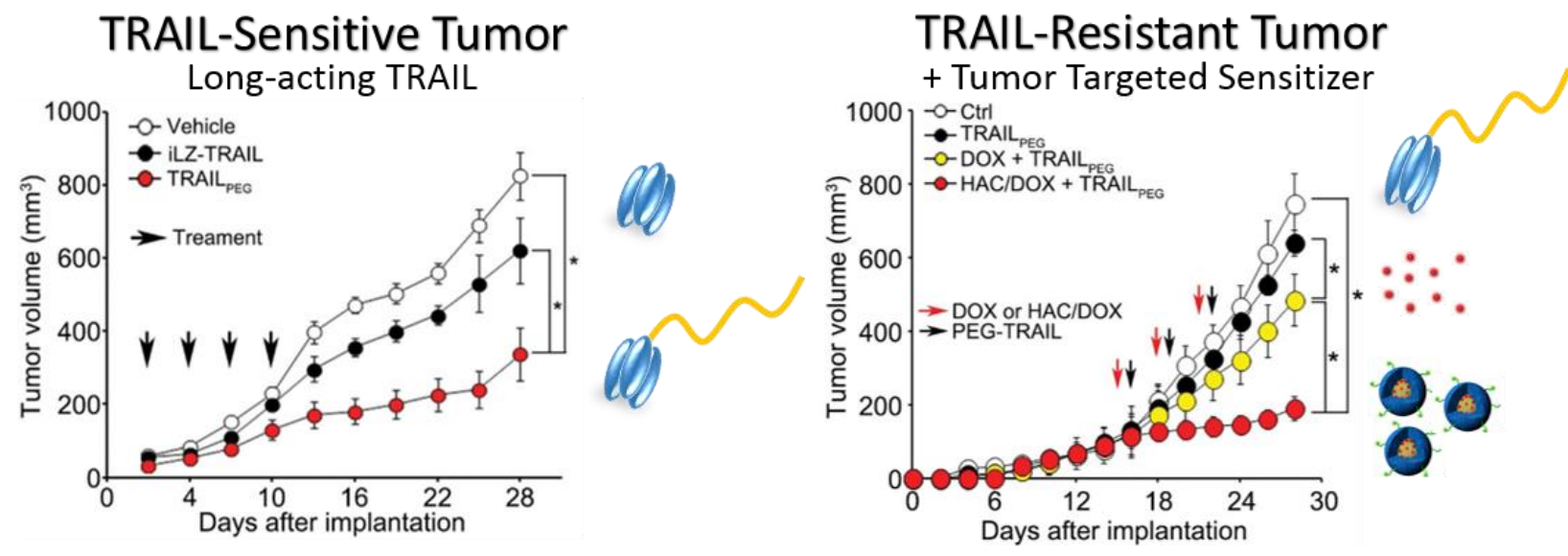